\title{
Design of Farmland Image Acquisition and Transmission System based on ZigBee
}

\author{
Zhongmin $\mathrm{Yao}^{1, \mathrm{a}}$, Hongyan Meng ${ }^{2, \mathrm{~b}}$, Fusan Guo ${ }^{1, \mathrm{c}}$ and Hongbo $\mathrm{Yu}^{1, \mathrm{~d}}$ \\ ${ }^{1}$ School of Communication and Electronic Engineering, Qiqihar University, \\ Qiqihar 161005, China \\ ${ }^{2}$ The First Hospital of Qiqihar, Qiqihar 161005, China

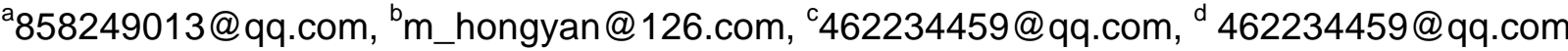

\begin{abstract}
Keywords: precision agriculture; farmland information; crop growth image; wireless sensor network; image acquisition sensor.

Abstract. Combine ZigBee and GPRS communication technology to design farmland image acquisition and transmission system. The system is composed of JN5139 ZigBee communication network and remote server monitoring software. Use JN5139 ZigBee chip to control the camera module with serial port to collect farmland image, and design program to realize reading and sending image packet to Coordinator Gateway on the application layer of ZigBee protocol stack. Image sensor node and Remote server software communicate with each other on Coordinator Gateway by GPRS module, and achieve farmland image acquisition and image data transmission. Test the system on the selected farmland, it can acquire and transmit farmland image successfully. The nodes' communication distance is $450 \mathrm{~m}$, and it costs about 150 s to transmitting a $320 * 240$ size JPGE image. So the system can meet the requirements of monitoring farmland environment.
\end{abstract}

\section{Introduction}

Farmland information acquisition is one of the key technologies of precision agriculture, and the crop growth image is one of essential basic farmland information [1]. Modern agriculture has put forward higher requirements for agricultural digitization, and Intelligent agriculture has become a research hotspot to enterprises and scientific research institutions. Crop growth images, field pest and weed monitoring are becoming their research focus. At home and abroad, sensing satellite and unmanned aerial are used to collect farmland image, but like greenhouse, because of the limitation of the cost and real-time, Crop growth images are collected by network cameras with network access port [2,3].

For existing communication technology, the wired communication network has the problems of difficult wiring, high complexity, high energy consumption and high cost. At the same time, mobile wireless communication and satellite communication depend on the communication operator, and there are difficulties in building network and Technology implementation. But ZigBee technology does not have these shortcomings, On the contrary, it has many advantages, like low power consumption, low cost, easy integration, easy networking. Although only $250 \mathrm{~kb} / \mathrm{s}$ (working at $2.4 \mathrm{GHz}$ ) rate, design API functions flexibly to meet the needs, that transmits the farmland image by Wireless sensor network $[4,5]$.

Combining with ZigBee and GPRS communication technology, Research Group has designed the system of farmland image acquisition and transmission. This system uses ZigBee JN5139 chip for networking, and image sensor nodes is composed of JN5139 chip and serial port camera module , it can collect farmland images and communicate data by ZigBee network. Coordinator gateway can communicate remote server with ZigBee network through the GPRS module, so remote server mobile transmits control commands to image sensor nodes by the GPRS module, and also can send farmland image data to the remote server. The remote server can receive and decode the image by socket programming, it also can display, store and analyze the images [6]. 


\section{System Structure}

The design of ZigBee farmland image acquisition and transmission system is mainly composed of ZigBee farmland image sensor network and remote server monitoring software. ZigBee network in the system is designed to a mesh network, it includes three functional modules: coordinator gateway, routing node and sensor node. JN5139 chip with GPRS as coordinator gateway is responsible for establishing ZigBee communication network and GPRS communication. Routing nodes can be multi-hop networking, expand the scope of networking, play repeater role; Terminal node with the serial port CMOS Camera, is responsible for acquiring farmland image acquisition and transmission image data packet. ZigBee coordinator gateway is communicated the remote server with GPRS module, and the remote server monitoring software can control the image sensor nodes to upload of the images, and can also decode, display and store the farmland images. The system structure is shown in Figure 1.

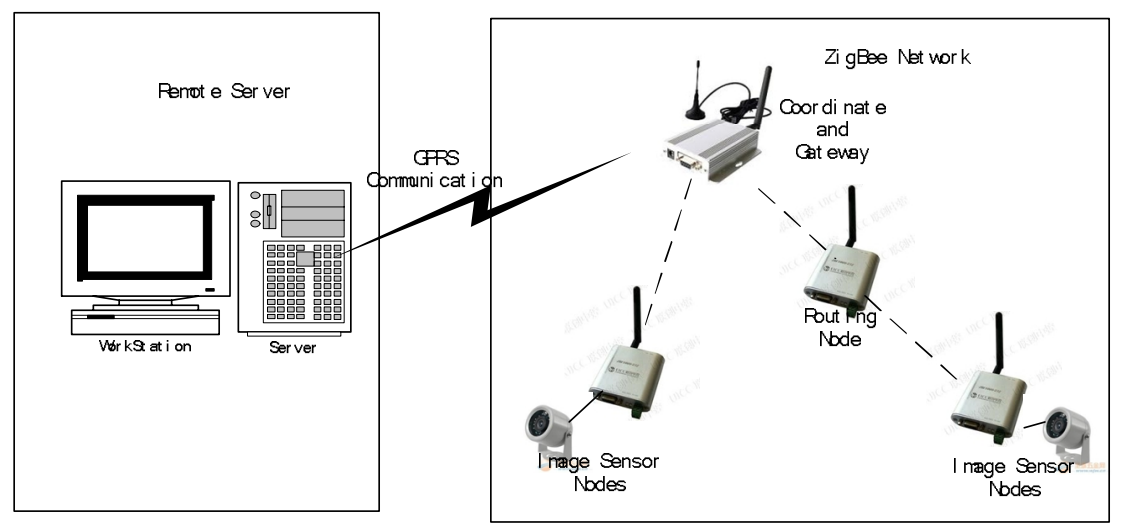

Fig. 1. System structure

\section{Hardware Design}

The hardware design mainly includes: the selection of ZigBee chip, image sensor node design and coordinator gateway design. TI's CC2430, CC2530 series chips and Jennic's JN5139, JN5148 series chips are used more welcomed in the country, but TI's CC2430, CC2530 series chip' kernel is an enhanced 80C51 micro-processing, having slow processing power; Jennic's JN5139, JN5148 series chip is a 32-bit RISC microprocessor, having strong data processing ability [7]. As the application environment of the subject is farmland image acquisition and transmission, the data volume of the image is relatively large, the transmission distance is as far as possible, in short ZigBee CPU is needed faster speed, as low as possible power consumption, so choose JN5139 chip as ZigBee Wireless communications core chip to solve the image sensor control and image data transmission problems. And then determine the key design points: image sensor nodes and coordinator gateway.

\section{Image sensor node design}

Image sensor node design is a focus of system hardware design, it mainly includes three aspects: collecting images of agricultural growth, transmitting the image data packet to the ZigBee coordinator gateway, extending PTZ interface to controls image sensor to acquire multi-directional Images. Jennic's JN5319 is selected as ZigBee network' core chip, to control image sensor and transmit image data wirelessly. The image sensor adopts PT08 image acquisition module designed and manufactured by Guangzhou Telecommunication Communication Technology Co., Ltd. It is an industrial image sensor, which integrates image acquisition, shooting control, data compression and serial transmission. The images output of PT08 are standard JPEG format, and the image pixel size and compression rate 
can be set by instruction, you can program control to read data packets from the storage space. The connection JN5139 chip and image sensor is shown in Figure 2.

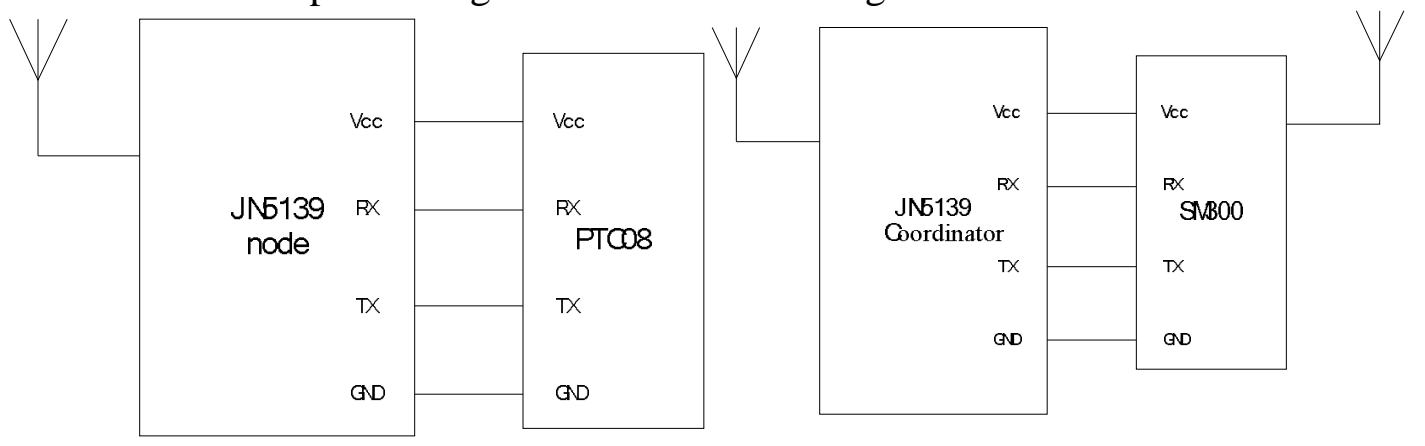

Fig. 2. End device and image sensor connection

Fig. 3. Coordinator gateway hardware structure

\section{Coordinator gateway}

The coordinator gateway is another important point of the hardware design. It mainly plays two roles in the system. On one hand, it is responsible for establishing the ZigBee wireless communication network, providing the sensor node scanning channel to join; on the other hand, re the image data received form image sensors is transmitted to the remote server-side monitoring program via the GPRS mobile network. This paper background is about the collection and transmission of farmland image, which requires ZigBee network to be convenient and low power consumption. So JNIC-JN5139 chip is chosen as the coordinator to set up ZigBee communication network. GPRS module selects SMCOM company's SM300. ZigBee network communicates the remote server with the GPRS module. Coordinator gateway hardware structure is shown in figure 3.

\section{Software design}

System software design includes: image acquisition and data transmission, GPRS software design and remote server monitoring software design. Image acquisition and data transmission are realized by the image sensor nodes. Design the corresponding program based on the hardware design of the image sensor node. New a project EndDevice.cbp under Jennic CodeBlocks, and this development environment is implemented by Jennic. GPRS software design is included GPRS data transmission and reception, which are achieved under the control of ZigBee coordinator processing chip. The project of Coordinate.cbp is also programmed under Jennic CodeBlocks, it can build a wireless sensor network and communicate with GPRS module. Remote server monitoring software can remotely send commands to control the camera initialization and taking pictures, and receive image data transmitted by GPRS module, then according to the image transmission protocol sort and compiling the image data, at last display the image on the remote server monitoring software interface, at the same time store and analysis the farmland images.

\section{Image acquisition and Data transmission}

The software design focuses of image sensor node are image sensor acquisition control and image data packet processing and transmission. After powering on the image sensor, it's system initializes, and searches and joins ZigBee network. In order to save energy consumption, the image sensor enters a 5-minute hibernation program after sending an image, and then waits for the remote server to wake up. At the image data transmission stage, reads the image data collected by the image sensor, and reads and sends the image data in groups.

\section{Image Data Acquisition}

In order to find and locate the image sensor nodes in the network, an integer variable for node label is pre-defined when the image sensor node program is designed and assigned it when the protocol stack is initialized. And define the control command communication protocol format between the remote server and the image sensor node in the system. The format is "\# image sensor node number $\$$ action instruction \&", the image sensor node number is determined according to the network scale, so accurately control the image sensor initialization and taking picture via the image sensor node number; 
there are two action instructions in data field of action instruction in the communication protocol format, action instructions-" 11 " is on behalf of the image sensor initialization; instructions " 21 " command image sensor to start taking pictures, get the image size, and transmit the image data by ZigBee network and GPRS mobile network.

When the image sensor node receives the communication command from the remote server or user terminal, at first image sensor node number in the communication protocol format will be compared with the local IDNum, if the condition is right, the program will determine the control command, then command -11 will Initialize the image sensor, else command - 21 will take picture. The Image sensor initialization process is as follows:

(1)Set the size of the camera;

(2)Set the compression rate of the picture;

(3)Set the baud rate.

The process of taking pictures is as follows:

(1)Clear image cache;

(2Take a picture;

(3) Read the length of the picture;

(4) Read a packet of image data [8].

We design the taking pictures process of Image sensor as figure 4.

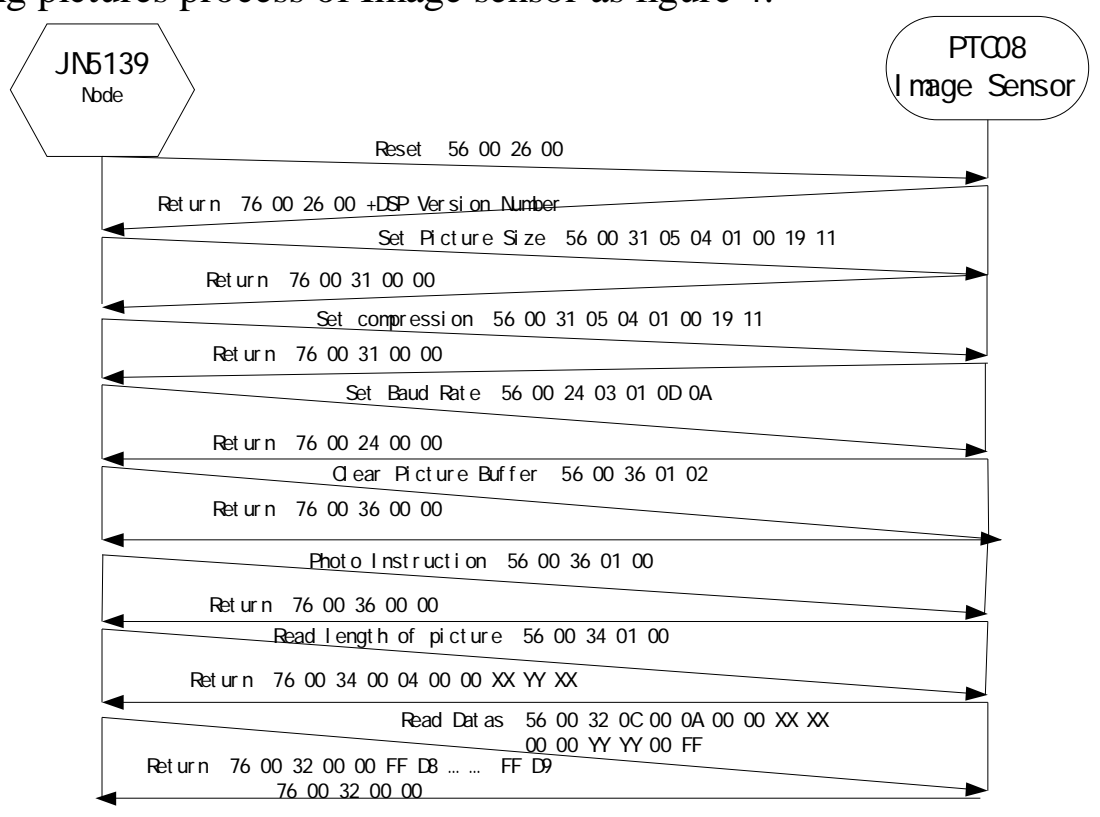

Fig. 4. Process of taking pictures

\section{Image data wireless transmission}

JN5139 chip's protocol stack is based on the ZigBee protocol stack of IEEE802.15.4, which stipulates that the physical layer only supports that the data frames of not more than 127 bytes is transmitted successfully, excluding application layer, network layer, MAC layer frame header and MAC layer frame Tail, the actual transmission application data of each frame does not exceed 89 bytes. Even if the image sensor set the image capture $320 * 240 \mathrm{JPEG}$ format, the size of the image are dozens of kilobits, which requires sending data packet on the application layer, the system stipulates that 80 bytes are sent every transmission $[9,10]$.

According to the length of the image data of each frame in the system, an image $\mathrm{X}$ is represented as group of data packets, $X=\{X 1, X 2, X 3, \ldots \ldots, X n\} . X 1$ is defined as the first frame of image data, its first 2 bytes is fixed to $0 \mathrm{xFF} 0 \mathrm{xD} 8 . \mathrm{Xn}$ is defined as the last frame of image data, its last 2 bytes are fixed to $0 x F F 0 x D 9.1,2,3, \ldots \ldots, n$ represents the image data packet sequence number[10].

ZigBee chip sends the command of taking a picture to the camera, when receiving the response instructions, then send reading the size of the picture command array , $\{0 \times 56,0 \times 00,0 \times 34,0 \times 01,0 \times 00\}$, 
the image sensor wil return the image length string , $\{76,00,34,00,04,00, X X, Y Y, X X, Y Y\}$, where the last four bytes are used to represent the size of the image, $\mathrm{XX}$ is the high bytes and $\mathrm{YY}$ is the low bytes. And then Calculate the value of the functions (Piclength\% 80 and Piclength / 80) to obtain image transmission times. According to the value, design the timer interrupt program to cycle two steps, one step read the data packet from the camera's Ram, another step Send image packets. Interrupt function body is a for loop. Timer interrupt program (vAppTick) implementation method is as follows :

if( flag_Read_Write ==FALSE)//One step read the image data packet.

\{

for $(\mathrm{j}=0 ; \mathrm{j}<\operatorname{sizeof}(\mathrm{cCommandBuffer}) ; \mathrm{j}++)$

$\{$ cCommandBuffer[j] $=0 ;\} / /$ clear the image buffer array

// Load the first address.

getphotocmd[8] $=($ ReadStep $*$ N_BYTE $>>8) \&$ 0xff; getphotocmd[9] = ReadStep * N_BYTE \& 0xff;

// Load the last address.

getphotocmd[12] $=($ N_BYTE $>>8) \&$ 0xff;

getphotocmd[13] = N_BYTE \& 0xff;

ZhongDuanFlag $=0$;

cam_write(getphotocmd,16);// Read the right data packet.

\}

if(flag_Read_Write==TRUE)// Another step send the data packet.

\{

for $(x=5 ; x<92 ; x++)$

\{

cCommandBuffer[x-5]=cCommandBuffer[x];//Truncate the useful fields.

\}

vSendData(u16DstAddres,80,cCommandBuffer); //Send data packets. //Reset the falg.

ZhongDuanFlag=0;

u8CommandTail $=0$;

// The image packet count flag is incremented by one.

ReadStep++;

\}

\section{GPRS software design}

GPRS module connected to the coordinator via the serial port, the coordinator receives the image data packets from the image sensor node. JN5139 chip controller control the GPRS module through the AT command, achieve to sending image data packets to a remote server, set a bridge between ZigBee network and Internet network. GPRS module software program design is included: sending and receiving data information. At first, GPRS module needs use "AT command" to connect with the remote sever, only when the communication link connection is successful, data communication is ok [11]. GPRS module SIM300 related configuration parameters are as follows:

$\mathrm{AT}+\mathrm{IPR}=115200 / /$ Set the SIM300 baud rate as $115200 \mathrm{~b} / \mathrm{s}$.

AT+CPIN? /* After the SIM card accessing, SIM card status information shows: + CPIN READY AT+CSQ.*/

// The received signal quality of the GPRS module is displayed as 1 to 30 .

AT+COPS? // Show SIM card service provider information.

$\mathrm{AT}+\mathrm{CLPORT}=$ " TCP" , " Port Number" // Set TCP connection protocol and Port number. AT +CIPSTART =" TCP" , “ IP" , " Port Number" // Initiated TCP connection.

$\mathrm{AT}+\mathrm{CIPSEND}=$ ? // Start to send data packets.

If the above settings are all normal, GPRS communication link is established, so the data packet can be sent between the sensor node and the remote server successfully. 


\section{Remote server monitoring software design}

Visual Studio2015 as the development environment, use C\# language to design the remote server management software. The system uses Socket to send and receive data, the application can send a camera command to the ZigBee image sensor node in accordance with the provisions of the communication protocol, while the image sensor nodes are real-time monitored Whether or not to send data [12]. Server software features mainly include: GPRS parameter settings and creating a server, ZigBee image sensor node management, database image management. SQL Server 2008 was used to build the database, and the corresponding server and client were written in $\mathrm{C} / \mathrm{S}$ architecture. The server not only can store the images in real time, but also access the farm image data through the client. After the server software runs, it needs to authenticate the user name and password, then enter the MDI parent form, select the "ZigBee image sensor node" option in the menu bar to complete the configuration of the image sensor node parameters; And in the option --"GPRS parameter settings and creating a server", you can complete the TCP/IP address and PROT port settings, and set the image acquisition mode for manual acquisition or timing acquisition; in" data image management "option, there are data storage, browsing history pictures, backup and emptying database. The flow chart of remote server monitor program is shown in Figure 5.

GPRS communication only can be connected to the public network, and the need for a fixed IP address, but the school computers access to Internet through the WAN miniport PPPOE without a fixed public IP address, and a rental server is not convenient and relatively expensive. Faced with this problem, our solution is to use peanut shell client (a software) to add a mapping, and solve the school network and the public network communication problem, so GPRS module can also communicate with the remote server.

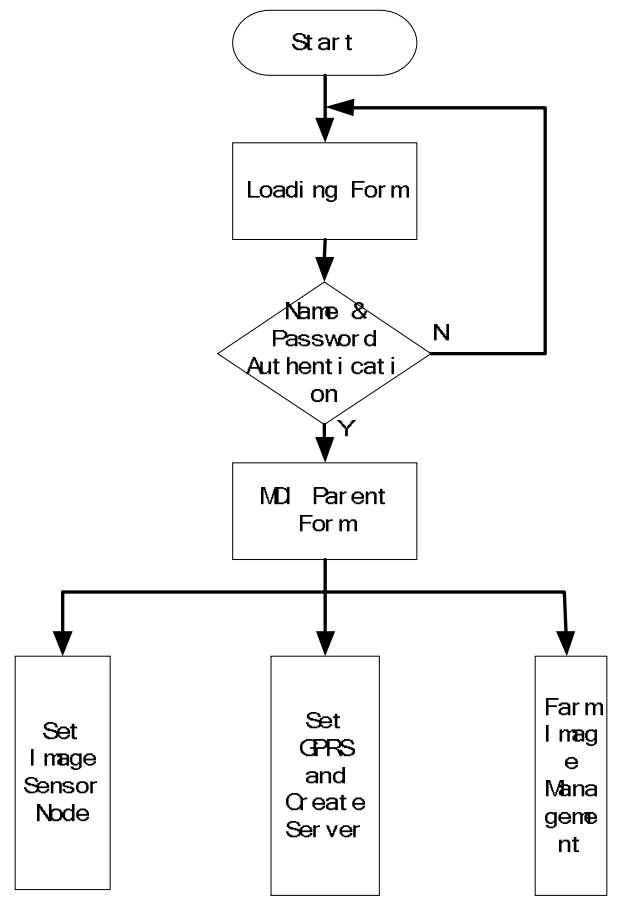

Fig. 5. Flow chart of remote server monitor program

\section{System testing}

Based on the system software and hardware design, the system were integrated and tested. The test environment was a $500 \mathrm{~m} * 500 \mathrm{~m}$ rice field with 3 image sensor nodes, 1 routing node and 1 coordinating Device, deployed into a mesh network structure. The results show that when the ZigBee communication equipment is placed over the rice, the communication distance is between $90 \mathrm{~m} \sim 100$ $\mathrm{m}$ when the high power is not switched on; the communication distance is between $450 \mathrm{~m} \sim 500 \mathrm{~m}$ when the high power mode is switched on, even in greenhouses and corn The transmission distance is 
$40 \mathrm{~m}$ and the packet loss rate is less than 0.092, which can meet the requirements of environment and system.

ZigBee theoretical rate is $250 \mathrm{~kb} / \mathrm{s}$, Sensor node and image sensor serial communication rate is $115200 \mathrm{bps}$, and China Mobile's GPRS rate is $171.2 \mathrm{~kb} / \mathrm{s}$, so system choose $115.2 \mathrm{kbps}$ as communication rate for the smooth communication. Based on the above data, the theoretical minimum transmission time of the image is about $320 * 240 * 8 / 115200=5.33 \mathrm{~s}$ when the image size is selected as $320 * 240$. Considering the time that ZigBee needs read data from the camera and the delay of transmission, Jn5139 protocol stack needs at least $320 * 240 / 80 * 2 * 10 / 1000=19.2 \mathrm{~s}$ taking and transmitting a picture, theoretically the minimum time of an image transmission is $19.2+5.33=24.5 \mathrm{~s}$. However the actual measured transmission time of the image is about $150 \mathrm{~s}$. Compared of theoretical transmission time and the actual transmission time difference, we analyze the main reasons, on one hand there are debug programs in the protocol stack, which does not shut down; on the other hand there is communication delay. The remote server monitoring software operation is shown in Figure 6.

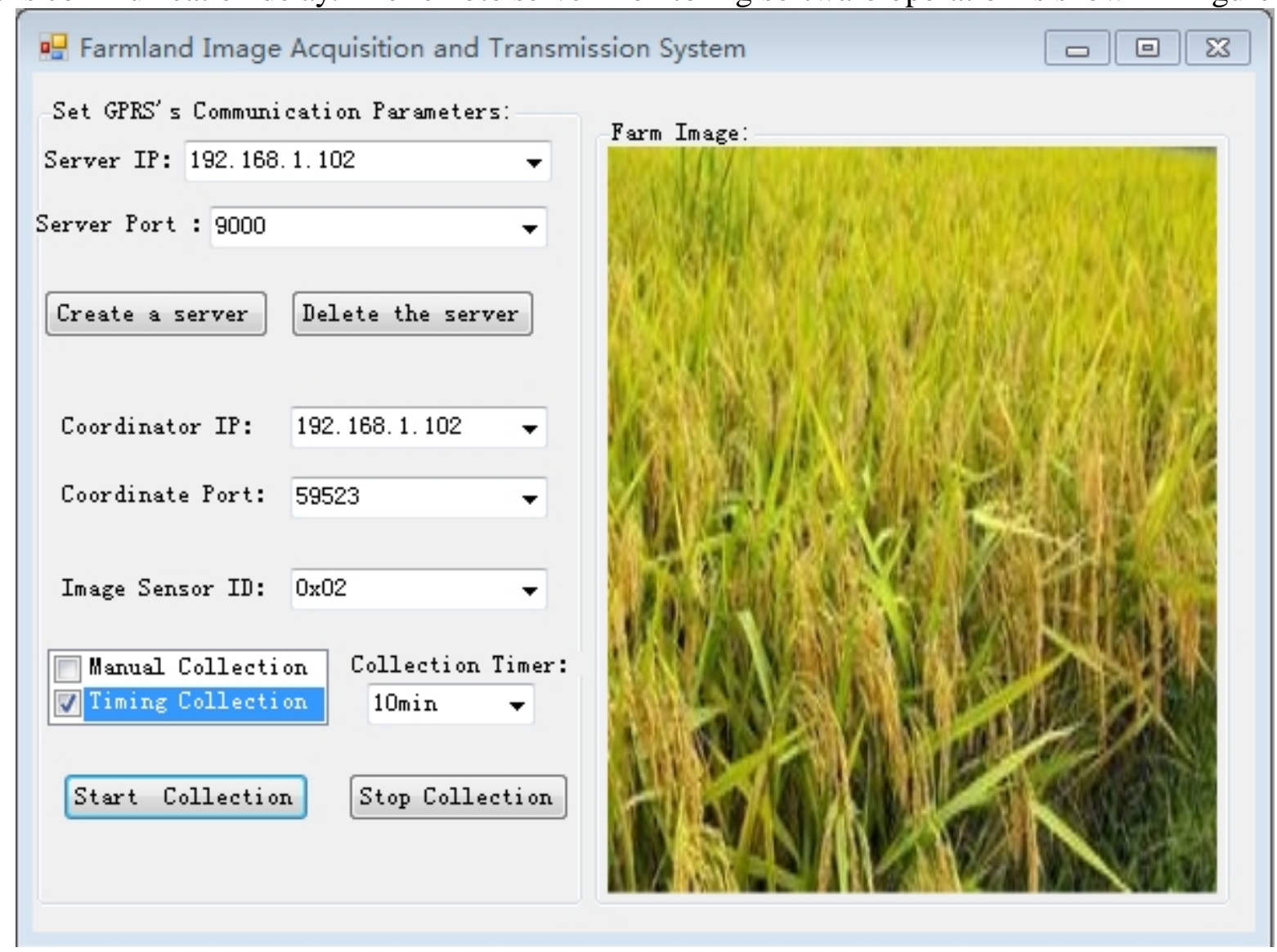

Fig. 6. Remote server monitoring software operation

\section{Conclusions}

"Precision Agriculture" needs to monitor the growth image of corps. The system design uses ZigBee and GPRS two kinds of communication technology, it can realize the farmland image acquisition and transmission. JN5139 chip is used to design ZigBee wireless sensor network, the image sensor node is composed of JN5139 chip and serial port camera module, and the coordinator gateway is composed of JN5139 chip and GPRS communication module. And also, the monitoring program on the remote server is written by C\# software. Based on the system hardware and software design, the system hardware and software are field-tested. In the field test, the farmland image acquisition function can be realized, and the image is transmitted to the remote server smoothly. In the experiment, the communication distance and packet loss rate of the sensor nodes are measured, the system theoretical time of transmitting an image is calculated and the system actually time of transmitting an image is measured, analyzed the difference between the two times. The research is farmland image acquisition 
and transmission based on ZigBee communication technology. It is not only applied to intelligent agriculture and smart home and security, but it also can be applied to environmental monitoring under high pollution, high radiation and high dangerous environment.

\section{Acknowledgements}

This work was financially supported by National Natural Science Foundation of China ( 51177072 ).

\section{References}

[1] Tao Ye. Research and Development of Filed Information Acquisition System in Precision Agriculture[D].Beijing: Chinese Academy of Agricultural Sciences,2006. (in Chinese)

[2] Jie Li, Jianshun Zhang, Hongyi Guan. The technology development of agricultural condition information Both Abroad and Home [J]. Science and Technology of Tianjin Agriculture and Forestry, 2000, 4(2): 36 - 39. (in Chinese).

[3] Yingjun Xiong, Mingxia Shen, Yuwen Sun. Design on System of Acquisition and Wireless Transmission for Farmland Image [J] .Chinese Journal of Agricultural Mechanization,2011,42(3):184-187. (in Chinese)

[4] Baronti P , Pillai P , Chook V , et al . Wireless sensor networks : A survey on the state of the art and the 802 . 15 . 4 and ZigBee standards [J] . Computer Communications , 2007, 30(7):1655-1695 .

[5] Pekhteryev G, Sah inoglu Z , Orlik P , et al . Image transmission over IEEE 802 . 15 . 4 and ZigBee networks[C].// IEEE International Symposium on Circuits and Systems , Kobe , Japan , 2005 , 4: 3539-3542.

[6] Wei Zhang, Yong He, Fei Liu, et al. Design of wireless sensor network node for field information collection[J]. Journal of Agricultural Mechanization Research,2015,01:135-139. (in Chinese)

[7] Zhaopeng Long. Design of Taxi Dispatching System Based on Wireless Communication Technology [D]. Qiqihar University, 2013. (in Chinese)

[8] Putai Communication Technology Co., Ltd. PTC08 serial port camera module instruction manual V1.07[P]. 2014.

[9] Zhiqiang Song, Xianzhong Zhou, Lei Wang. Research on Wireless Image Transmission Based on ZigBee[J]. Computer Applications and Software, 2012,29(7),1-2,5. (in Chinese) 
[10] Chunjiang Zhao, Lihua Qu, Ming Chen. Design of ZigBee-based Greenhouse Environment Monitoring Image Sensor Node[J]. Transactions of the Chinese Society for Agricultural Machinery,2012,43(11):192-196. (in Chinese)

[11] SIMcom Technology Co., Ltd. SIM300 instruction manual [P], 2005.

[12] Jun Ma. Network Application Programming [M]. People Post Press, 2010(2). (in Chinese) 